Vázquez Romero, I.M. y Blanco-Blanco, A. (2019). Factores sociocognitivos asociados a la elección de estudiós científico-matemáticos. Un análisis diferencial por sexo y curso en la Educación Secundaria. Revista de Investigación Educativa, 37(1), 269-286.

DOI: http://dx.doi.org/10.6018/rie.37.1.303531

\title{
Factores sociocognitivos asociados a la elección de estudios científico-matemáticos. Un análisis diferencial por sexo y curso en la Educación Secundaria
}

\author{
Sociocognitive factors associated with the choice \\ of scientific-mathematical studies. A differential analysis by genre \\ and course in Secondary Education
}

\author{
Isabel Ma Vázquez Romero y Ángeles Blanco-Blanco* \\ Universidad Complutense de Madrid
}

\begin{abstract}
Resumen
El estudio se inserta en la investigación orientada a entender las trayectorias de elección vocacional de los jóvenes en los campos profesionales de la ciencia, la tecnología, la ingeniería y las matemáticas (STEM). Dada la constatada brecha de género, el análisis se centra en analizar posibles diferencias entre alumnos y alumnas a lo largo de distintos cursos de educación secundaria en un conjunto de variables sociocognitivas con una relevancia bien establecida en el desarrollo vocacional. Se utiliza como marco la teoría cognitivo social del desarrollo de la carrera (SCCT). En el estudio participaron 1465 estudiantes españoles. Todos ellos completaron medidas de autoeficacia, expectativas de resultados, intereses, aspiraciones ocupacionales y apoyos y barreras sociales percibidas en la elección de estudios científico-matemáticos. Se aplicaron pruebas no paramétricas y se estimaron medidas del tamaño del efecto para las comparaciones por género y curso. Se identificaron diferencias significativas a favor de los varones, en general de baja magnitud, en todas las variables salvo en las aspiraciones ocupacionales. Igualmente se

Correspondencia: Ángeles Blanco Blanco, ablancob@ucm.es. Departamento de Métodos de Investigación y Diagnóstico en Educación. Universidad Complutense de Madrid.

* Este estudio fue diseñado durante una estancia de investigación en la Universidad de Maryland (USA) realizada por la autora de contacto con la financiación del Ministerio de Economía y Competitividad (Programa Nacional de Movilidad de Recursos Humanos, Programa José Castillejo), bajo la supervisión del profesor Robert W. Lent.
\end{abstract}


identificó una tendencia significativa a presentar menores promedios en todas las variables a medida que se avanza en la secundaria. Sin embargo, este patrón de resultados presentó aspectos diferenciales al considerar el curso y/o la modalidad de bachillerato. Los resultados se discuten en el contexto de la investigación previa y se sugieren líneas de trabajo futuro desde el punto de vista de la investigación y de la intervención educativa.

Palabras clave: teoría cognitivo social del desarrollo de la carrera; educación STEM; estudiantes de educación secundaria; diferencias de género.

\section{Abstract}

This study is part of the research aimed at understanding vocational choice trajectories of students in professional areas related to science, technology, engineering and mathematics (STEM). Due to the well-known gender gap, the study is focused on analyzing possible differences between women and men in several socio-cognitive variables with a well-established relevance in vocational development. Differences along different grades of secondary education are also discussed. Social Cognitive Career Theory (SCCT) is used as a framework. In this study 1,465 high school Spanish students were involved. All of them were evaluated for self-efficacy, outcome expectations, interests, support, occupational aspirations and perceived social barriers when starting careers in the science/mathematics area. Non-parametric statistic tests were applied as well as measures of the effect size comparing by gender and course. Significant differences were found in favor of males, usually of low magnitude, in all the variables analyzed with the exception of those concerning the occupational aspirations. Likewise, a significant tendency was identified to present lower averages in all the variables as it progresses in secondary school. However, the general pattern of results showed differentiating aspects when considering a course and/or a kind of high school curriculum. The results are discussed in the context of the previous research on this topic and future lines of work are suggested from the point of view of the research and also educational intervention.

Keywords: social cognitive career theory; STEM education; secondary education students; gender differences.

\section{Introducción}

En el proceso de toma de decisiones académicas y profesionales ser varón o mujer parece seguir siendo un factor determinante. En España son abundantes los trabajos que han informado de que los chicos tienden a elegir estudios y opciones laborales científicas, matemáticas o técnicas en mayor medida que las chicas (Canto, 2000; Vázquez \& Manassero, 2008, 2009; Rodríguez, Inda y Peña, 2014; Rodríguez, Peña \& Inda, 2012). Por el contrario, las chicas optan por opciones humanísticas y sociales (Rodríguez, Torio \& Fernández, 2006; Santana, Feliciano \& Jiménez, 2012). Este patrón parece común en otros países, lo que ha promovido un área de investigación específica centrada en los factores explicativos de la brecha de género presente en los procesos de selección de carreras en el ámbito de ciencias, tecnología, ingeniería y matemáticas, más conocido como STEM (Vázquez \& Manassero, 2015). Y es que efectivamente la presencia diferencial de mujeres y varones en estos campos profesionales está ampliamente documentada (Alloza, Anghel, Dolado, De la Rica \& Sánchez de Madariaga, 2011). 
El fenómeno debe entenderse desde una problemática más general: la falta de atractivo que los cursos STEM despiertan en los estudiantes españoles, globalmente considerados, antes de ingresar en la universidad (Vázquez \& Manassero, 2008). Una vez más la crisis general de vocaciones en el área STEM parece registrarse en buena parte de los países occidentales (Organización para la Cooperación y el Desarrollo Económicos [OECD], 2008). Esto tiene un claro reflejo en uno de los objetivos del programa Horizonte 2020 de la Unión Europea: aumentar las tasas de egresados en el ámbito STEM en el periodo 2014-2020 (Centro para el Desarrollo Técnico Industrial [CDTI], 2014). Una visión detallada de este reto desde la perspectiva de género, por tanto, subraya adicionalmente la necesidad de corregir la pérdida de capital humano asociada a la desigual incorporación de las mujeres y de su desarrollo profesional en los ámbitos científico-técnicos.

En términos generales puede decirse que, una vez superada la hipótesis del desempeño y el rendimiento diferenciales entre sexos, como principal factor determinante del patrón desigual en la elección de estudios, la investigación se ha concentrado en factores de tipo psicológico, sociocultural y contextual. Es el caso de la aplicación al estudio de las trayectorias de elección en el área STEM de la Teoría de la ExpectanciaValor (véase Wang \& Degol, 2013) o de la Teoría Cognitivo Social del desarrollo de la carrera (SCCT) (Lent, Brown \& Hackett, 1994, 2000).

La evidencia sobre la utilidad explicativa de la SCCT es notable (véase la revisión meta-analítica de Sheu et al., 2010). Pero su ámbito de aplicación más desarrollado es el científico-matemático, tecnológico y vinculado a la ingeniería, donde sus resultados la han convertido en uno de los principales marcos usados para el análisis de los procesos de selección y persistencia en el área STEM (Wang, 2013).

El núcleo de la SCCT está constituido por: la autoeficacia (creencias sobre nuestra habilidad para realizar con éxito tareas de un dominio específico), las expectativas de resultado (auto-valoración sobre los resultados que obtendremos al realizar una elección académica), los intereses (preferencias por determinas actividades académicas) y las metas (objetivos o intenciones de implicación en actividades académicas), que conducirán a la implicación en una opción académico-profesional. En torno a este núcleo se organizan otros factores de tipo personal (p.e. sexo) y contextual. Entre éstos últimos la investigación ha prestado especial interés a los apoyos y barreras sociales percibidas por los sujetos en los procesos de elección de estudios u opciones académico-profesionales. Este modelo teórico postula que las relaciones hipotetizadas entre los constructos descritos no están moderadas en razón del sexo, y que por tanto su capacidad explicativa no varía entre varones y mujeres, con independencia de que éstos difieran en el grado en el que presentan expectativas positivas, intereses, fijación de metas o cualesquiera otra de las variables consideradas en el mismo.

La SCCT ha sido ampliamente usada en la investigación empírica principalmente con muestras de estudiantes norteamericanos universitarios (p.e. entre otros muchos Byars-Winston, Estrada, Howard, Davis \& Zalapa, 2010; Lent et al., 2001; Lent, Sheu, Gloster \& Wilkins, 2010; Lent et al., 2008) y de Secundaria (p.e. Flores \& O'brien, 2002; Fouad \& Smith, 1996; Garriot, Flores \& Martens, 2013; Navarro, Flores \& Worthington, 2007), incluyendo frecuentemente análisis específicos para ambos sexos. Globalmente 
se ha mostrado invariante, esto es, útil para explicar los procesos de desarrollo vocacional de varones y mujeres. Pero la investigación también ha permitido identificar diferencias entre varones y mujeres en varios constructos, lo que puede contribuir a la explicación de los patrones diferenciales de elección. Navarro et al. (2007) y O’Brien, Martínez-Pons y Kopola (1999) hallaron menores niveles de autoeficacia en chicas que en sus pares varones. También se ha informado de un menor interés de las chicas en el área STEM (p.e. Turner \& Lapan, 2005) y expectativas de resultados menos positivas (Fouad \& Smith, 1996). Por el contario, Navarro et al. (2007) encontraron que las chicas presentaban niveles superiores en apoyos y barreras sociales.

La SCCT se ha demostrado también útil en diversos contextos culturales (Sheu \& Bordon, 2017) y en concreto ha sido evaluada positivamente con muestras españolas en el área STEM, tanto en el nivel universitario (Blanco, 2011; Rodríguez, Inda \& Peña, 2015) como en Secundaria (Rodríguez, Inda \& Fernández, 2016).

El estudio de Rodríguez et al. (2016) se centró en el ámbito de la Tecnología y evaluó con resultados satisfactorios, en una amplia muestra de estudiantes de Secundaria, el ajuste de un modelo cognitivo social. Dicho modelo incorporó las variables: autoeficacia, expectativas de resultado, intereses, apoyos y barreras sociales percibidas, estado emocional y actitudes de rol de género. El modelo explicó una proporción sustancial de la varianza de los intereses y se mostró invariante para varones y mujeres, conforme a lo hipotetizado. Sin embargo, y frente a la mayor parte de los resultados de la investigación previa, no se encontraron diferencias entre varones y mujeres en autoeficacia, expectativas de resultado, intereses o apoyo social percibido.

El trabajo anterior constituye hasta la fecha el único antecedente de aplicación en la Secundaria de la SCCT al análisis del ámbito STEM en España, presentando resultados prometedores y sugiriendo cuestiones adicionales pendientes de respuesta. Entre ellas queremos destacar la ausencia de diferencias entre varones y mujeres en los factores sociocognitivos evaluados. Si bien, como los propios autores sugieren, ello puede estar informando de un cambio de tendencia real, también pudiera ser un resultado artificial asociado a los instrumentos de medida o dependiente del sub-ámbito específico de referencia, el tecnológico. Adicionalmente, puesto que en el estudio no se incorporó ninguna variable de la dimensión de metas, no se evaluaron posibles diferencias en los objetivos o aspiraciones ocupacionales entre varones y mujeres. Finalmente, el análisis diferencial se condujo con las submuestras globales de varones y mujeres, lo que no permitió considerar factores intervinientes, tales como las modalidades cursadas por alumnos y alumnas. El presente estudio trata de avanzar en estas cuestiones pendientes.

Además, en perspectiva longitudinal, las trayectorias de elección también parecen afectadas para varones y mujeres por lo que se ha calificado de paulatina depresión actitudinal en el ámbito científico-matemático (Vázquez \& Manassero, 2005), entendida como una visión progresivamente más negativa de la ciencia a medida que los estudiantes avanzan en la escolarización. Así, el interés por las matemáticas, su utilidad de cara al futuro, la competencia percibida para el aprendizaje y el logro decrecen significativamente desde los primeros cursos de Primaria hasta los últimos de Secundaria (Nuñez et al., 2005), siendo un fenómeno extensivo al área de ciencias 
y más acusado para las chicas durante su adolescencia, pudiendo estar relacionado con la creciente aceptación del rol femenino de género (Vázquez \& Manassero, 2008). Si bien esta tendencia no es exclusiva de este ámbito, puesto que la actitud hacia la escuela en general se ve disminuida, es especialmente acusado en las materias científicas, técnicas y matemáticas (Hidalgo, Maroto \& Palacios, 2004). Cabe por tanto preguntarse si dicho deterioro afecta al conjunto de las variables incluidas en el modelo SCCT y en qué grado, y si lo hace para ambos sexos. Esta cuestión no se ha investigado previamente en la educación secundaria española, por lo que su análisis podría contribuir a aumentar la comprensión de las trayectorias de elección en el área STEM.

\section{Método}

\section{Objetivos}

En el contexto de los antecedentes expuestos, los objetivos principales del presente estudio son los siguientes:

- Realizar un análisis diferencial entre sexos en alumnado de educación secundaria para todas las variables centrales del modelo cognitivo social del desarrollo de la carrera en el ámbito científico-matemático, incluyendo particularmente las aspiraciones ocupacionales.

- Estudiar la modalidad cursada en Bachillerato como variable relevante en el análisis de las potenciales diferencias asociadas a las variables consideradas.

- Analizar las posibles diferencias en los factores sociocognitivos considerados a lo largo de los distintos cursos de la educación secundaria.

Adicionalmente, como un objetivo instrumental previo orientado a determinar la sensibilidad de los instrumentos de medida usados, en términos de capacidad para discriminar entre el alumnado que optó o no por cursos con contenido científico-matemático, en el estudio también se pretende verificar la siguiente hipótesis: el alumnado de Bachillerato de Ciencias y Tecnología tendrá mayores puntuaciones promedio que el de Humanidades y Ciencias Sociales en todas la variables medidas.

\section{Población y muestra}

La muestra estuvo formada por un total de 1465 estudiantes (53\% mujeres y 47\% varones), de entre 14 y 18 años, matriculados en Educación Secundaria Obligatoria -ESO (tercero $28 \%$, cuarto $25 \%$ ) y Bachillerato (primero $34 \%$, segundo $13 \%$ ), incluyendo las modalidades Científico-Tecnológica (49\%) y de Humanidades y Ciencias Sociales (51\%). Los sujetos procedían de 8 centros (7 públicos y 1 concertado) de varias ciudades del centro de España: Madrid (22\%), Alcobendas (10\%), Cuenca (42\%), Albacete (14\%) y Guadalajara (12\%). 


\section{Instrumentos}

Las variables fueron medidas con cinco escalas sociocognitivas de formato tipoLikert y 7 puntos de respuesta, adaptadas y validadas por Blanco-Blanco, Casas y Mafokozi (2016).

- Autoeficacia científico-matemática. Compuesta por 12 ítems que evalúan el grado en el que los estudiantes creen que pueden llevar a cabo adecuadamente una serie de tareas relacionadas con las ciencias y las matemáticas (p.e. Sacar un sobresaliente en Matemáticas este curso). Presenta una estructura trifactorial: competencia percibida para obtener altas calificaciones en asignaturas científico-matemáticas, para realizar actividades diversas relacionadas con las Matemáticas y para realizar actividades diversas relacionadas con la Ciencia (GFI=.99; RMSR=.05). El coeficiente de fiabilidad es $\alpha=.86$.

- Expectativas de resultado en el área ocupacional científico-matemática. 9 ítems que expresan resultados positivos que podrían derivarse de una elección académicoprofesional en el ámbito científico-matemático (p.e. Aumentar mi propia autoestima). Presenta una estructura unifactorial (GFI=.99; RMSR=.06) y un coeficiente $\alpha$ de 91 .

- Intereses científico- matemáticos. 16 ítems que evalúan el interés de los estudiantes en la realización de diversas tareas vinculadas a las ciencias y las matemáticas (p.e. Visitar un museo de Ciencias). Se pueden diferenciar tres factores: interés por las actividades relacionadas con las Matemáticas, con la Ciencia y con la Tecnología (GFI=.99; RMSR =.05)). Presenta una fiabilidad de .89 .

- Aspiraciones u objetivos ocupacionales en el área científico-matemática. Inventario de 10 profesiones brevemente descritas (p.e. Astrónomo). Los estudiantes expresan cómo de seriamente considerarían cada una como su posible profesión. Presenta una estructura unifactorial (GFI=.98; RMSR=.08) y un coeficiente de fiabilidad igual a .88.

- Apoyos y barreras sociales percibidas en la elección de ocupaciones científico-matemáticas. 8 ítems sobre los apoyos y barreras percibidos en la decisión de acceder a una profesión que implicara habilidades científicas o matemáticas (p.e. Sentiría que los miembros de mi familia apoyan esta decisión). Se trata de una escala bifactorial $(\mathrm{GFI}=1.00$; RMSR=.03) con un coeficiente de fiabilidad igual a .76. La puntuación total se obtiene invirtiendo los ítems negativos que expresan barreras, por lo que una mayor puntuación indica un mayor nivel de apoyo social percibido.

\section{Procedimiento de recogida y análisis de datos}

Los estudiantes cumplimentaron las escalas como parte de un instrumento más amplio. La aplicación fue colectiva, llevada a cabo en horario lectivo y supervisada. Se obtuvieron los permisos necesarios de responsables escolares, familias y estudiantes.

Se obtuvo la puntuación total en cada una de las escalas, obtenida como la media de las respuestas a cada ítem. Para comparar los niveles promedio de las variables sociocognitivas a lo largo de los distintos grupos conformados por las variables sexo y curso, se usaron pruebas no paramétricas de contraste de hipótesis. Esta decisión se tomó tras verificar alguna desviación en la normalidad de las distribuciones y 
especialmente tras detectar la falta de homocedasticidad asociada a una parte de los contrastes. Atendiendo a esta circunstancia y con el fin de proporcionar un tratamiento común a todos los contrastes, se emplearon las alternativas clásicas no paramétricas: U de Mann-Whitney y $\mathrm{H}$ de Kruskal-Wallis.

Además, se obtuvieron medidas de tamaño del efecto y se realizaron pruebas post hoc de comparación por pares. En ambos casos se seleccionaron procedimientos ajustados al marco no paramétrico del análisis, siguiendo las sugerencias de la literatura especializada (véase p.e. Corder \& Foreman, 2009; Rovai, Baker \& Ponton, 2014). Concretamente, para estimar el tamaño del efecto se obtuvo el estadístico mostrado en la fórmula [1].

$$
r=\frac{|z|}{\sqrt{N}} \quad[1]
$$

Para la interpretación de este estadístico se hizo uso de los criterios clásicos ofrecidos por Cohen (1988). Valores por debajo de .30 son indicativos de un efecto pequeño, entre .30 y .50 valorados moderados y por encima de este valor puede considerarse alto.

Se hizo uso del ajuste de Bonferroni para el control de la tasa de error tipo I en las comparaciones múltiples.

\section{Resultados}

\section{Análisis preliminar de la sensibilidad de las medidas: diferencias en las variables sociocognitivas entre modalidades de Bachillerato}

Como paso previo al resto de los análisis, se compararon los promedios obtenidos en las variables en función de las dos modalidades de Bachillerato (ver tabla 1).

Conforme a lo esperado, el alumnado de Ciencias y Tecnología presentó en todas las medidas niveles promedio estadísticamente superiores que el de Humanidades y Ciencias Sociales. Atendiendo a las medidas de tamaño del efecto obtenidas, las diferencias halladas pueden ser calificadas como de magnitud media.

\section{Análisis de las diferencias en las variables sociocognitivas asociadas al sexo}

La tabla 2 presenta los resultados de los contrastes de las hipótesis relativas a la existencia de posibles diferencias entre sexos en los niveles promedio de autoeficacia, expectativas de resultado, intereses, aspiraciones ocupacionales y apoyos y barreras sociales percibidas.

Ambos sexos presentaron diferencias significativas entre sí en cuatro de las cinco variables consideradas: autoeficacia, intereses, expectativas de resultado y apoyos y barreras sociales. Los chicos presentaron un promedio significativamente más alto que las chicas en autoeficacia, expectativas de resultado e intereses. Tales diferencias son de baja magnitud. Sin embargo, en los apoyos y barreras sociales las chicas mostraron un promedio significativamente mayor, aunque de igual modo la diferencia es de baja magnitud. Por otro lado, no se hallaron diferencias significativas entre varones y mujeres en las aspiraciones ocupaciones relativas al área científico-matemática. 
Tabla 1

Comparación de promedios obtenidos en las modalidades de bachillerato

\begin{tabular}{lcccc}
\hline & Mediana & $\mathrm{U}$ & $\mathrm{p}$ & $r$ \\
\hline Autoeficacia & & 31013.00 & .000 & 0.43 \\
$\quad$ Humanidades y Ciencias Sociales & 3.83 & & & \\
$\quad$ Ciencias y Tecnología & 4.92 & & & 0.28 \\
\hline Expectativas de resultado & & 41843.00 & .000 & \\
$\quad$ Humanidades y Ciencias Sociales & 4.78 & & & \\
$\quad$ Ciencias y Tecnología & 5.44 & & .000 & 0.54 \\
\hline Intereses & 3.44 & & & \\
$\quad$ Humanidades y Ciencias Sociales & 3.94 & & .000 & 0.39 \\
$\quad$ Ciencias y Tecnología & & 33526.00 & & \\
\hline Aspiraciones ocupacionales & 2.20 & & & \\
$\quad$ Humanidades y Ciencias Sociales & 3.30 & & & \\
$\quad$ Ciencias y Tecnología & & 34178.00 & & \\
\hline Apoyos y barreras sociales & 5.25 & & & \\
$\quad$ Humanidades y Ciencias Sociales & 6.12 & & & \\
$\quad$ Ciencias y Tecnología & & & & \\
\hline
\end{tabular}

Nota. Humanidades y Ciencias Sociales: N=359; Ciencias y Tecnología: N=343.

Tabla 2

Test global de hipótesis sobre diferencias entre varones y mujeres

\begin{tabular}{lcccc}
\hline & Mediana & $\mathrm{U}$ & $\mathrm{p}$ & $r^{a}$ \\
\hline Autoeficacia & & 234237.50 & .000 & .13 \\
$\quad$ Mujer & 4.33 & & & \\
$\quad$ Varón & 4.67 & & & .08 \\
\hline Expectativas de resultado & 5.33 & & & \\
$\quad$ Mujer & 5.44 & & & \\
$\quad$ Varón & & 2234347.00 & .003 & .16 \\
\hline Intereses & 4.25 & & .000 & \\
$\quad$ Mujer & 4.69 & & & \\
$\quad$ Varón & & 273264.50 & .784 & \\
\hline Aspiraciones ocupacionales & 3.10 & & & \\
$\quad$ Mujer & 3.10 & & & \\
$\quad$ Varón & & 235579.00 & .000 & \\
\hline Apoyos y barreras sociales & 6.00 & & & \\
$\quad$ Mujer & 5.75 & & & \\
$\quad$ Varón & & & & \\
\hline
\end{tabular}

Nota. Mujer: N=785; Varón: N=702. a Obtenido para las diferencias significativas.

A continuación, interesaba determinar si este patrón hallado para el conjunto de la muestra se repetía con independencia del curso y la modalidad de bachillerato (ver tabla 3). 
Tabla 3

Diferencias entre sexos por curso y modalidad. Tamaño del efecto y grupo que presenta mayor puntuación

\begin{tabular}{|c|c|c|c|c|c|}
\hline & $\begin{array}{l}\text { Mediana } \\
\text { mujeres }^{\text {a }}\end{array}$ & $\begin{array}{l}\text { Mediana } \\
\text { varones }^{\mathrm{a}}\end{array}$ & $\mathrm{U}$ & $\mathrm{p}$ & $r^{b}$ \\
\hline \multicolumn{6}{|l|}{ Autoeficacia } \\
\hline $3^{\circ} \mathrm{ESO}$ & 4.58 & $4.81^{*}$ & 17861.50 & .001 & 0.16 \\
\hline $4^{\mathrm{o}} \mathrm{ESO}$ & 4.37 & $4.62^{*}$ & 14284.50 & .016 & 0.13 \\
\hline $1^{\varrho}$ Ciencias y Tecnología & 4.96 & 4.92 & 7958.00 & .941 & - \\
\hline $1^{\circ}$ Humanidades y Ciencias Sociales & 3.67 & $3.96^{*}$ & 6618.00 & .027 & 0.14 \\
\hline $2^{\circ}$ Ciencias y Tecnología & 4.85 & 4.75 & 946.00 & .960 & - \\
\hline $2^{\circ}$ Humanidades y Ciencias Sociales & 3.83 & $4.25^{*}$ & 865.50 & .002 & 0.29 \\
\hline \multicolumn{6}{|l|}{ Expectativas de resultado } \\
\hline $3^{\circ} \mathrm{ESO}$ & 5.55 & $5.78^{*}$ & 18898.50 & .014 & 0.11 \\
\hline $4^{\mathrm{o}} \mathrm{ESO}$ & 5.33 & 5.44 & 15192.50 & .131 & - \\
\hline 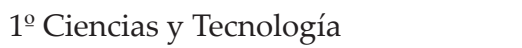 & 5.55 & 5.55 & 7919.00 & .888 & - \\
\hline $1^{\circ}$ Humanidades y Ciencias Sociales & 4.67 & 4.94 & 7519.00 & .514 & - \\
\hline $2^{\circ}$ Ciencias y Tecnología & 5.28 & 5.44 & 850.00 & .396 & - \\
\hline $2^{\circ}$ Humanidades y Ciencias Sociales & 4.67 & 4.78 & 1311.50 & .892 & - \\
\hline \multicolumn{6}{|l|}{ Intereses } \\
\hline $3^{\circ} \mathrm{ESO}$ & 4.56 & $4.78^{*}$ & 18556.00 & .006 & 0.13 \\
\hline $4^{\mathrm{o}} \mathrm{ESO}$ & 4.25 & $4.81^{*}$ & 12198.50 & .000 & 0.23 \\
\hline $1^{\circ}$ Ciencias y Tecnología & 4.84 & 5.00 & 7423.50 & .321 & - \\
\hline $1^{\circ}$ Humanidades y Ciencias Sociales & 3.31 & $3.69^{*}$ & 6486.50 & .015 & 0.15 \\
\hline 2 Ciencias y Tecnología & 4.47 & $5.16^{*}$ & 712.00 & .046 & 0.21 \\
\hline $2^{\mathrm{O}}$ Humanidades y Ciencias Sociales & 3.19 & 3.50 & 1036.50 & .055 & - \\
\hline \multicolumn{6}{|l|}{ Aspiraciones ocupacionales } \\
\hline $3^{\mathrm{o}} \mathrm{ESO}$ & 3.50 & 3.60 & 21718.50 & .857 & - \\
\hline $4^{\circ} \mathrm{ESO}$ & 3.20 & 3.15 & 16318.50 & .691 & - \\
\hline $1^{\circ}$ Ciencias y Tecnología & $3.65^{*}$ & 3.20 & 6432.50 & .007 & 0.17 \\
\hline $1^{\underline{o}}$ Humanidades y Ciencias Sociales & 2.20 & 2.20 & 7816.50 & .890 & - \\
\hline $2^{\circ}$ Ciencias y Tecnología & 3.20 & 3.35 & 830.50 & .312 & - \\
\hline $2^{\circ}$ Humanidades y Ciencias Sociales & 1.80 & 2.60 & 1088.50 & .113 & - \\
\hline \multicolumn{6}{|l|}{ Apoyos y barreras sociales } \\
\hline $3^{\circ} \mathrm{ESO}$ & 5.87 & 6.00 & 21255.50 & .579 & - \\
\hline $4^{\mathrm{o}} \mathrm{ESO}$ & $5.87^{*}$ & 5.50 & 13401.00 & .001 & 0.17 \\
\hline $1^{\circ}$ Ciencias y Tecnología & $6.31^{*}$ & 6.00 & 6149.50 & .001 & 0.20 \\
\hline $1^{\mathrm{o}}$ Humanidades y Ciencias Sociales & $5.62^{*}$ & 4.94 & 5914.50 & .001 & 0.15 \\
\hline $2^{2}$ Ciencias y Tecnología & 6.44 & 6.12 & 831.00 & .312 & - \\
\hline $2^{\underline{o}}$ Humanidades y Ciencias Sociales & 5.50 & 4.75 & 1069.50 & .088 & - \\
\hline
\end{tabular}

Nota. $3^{\circ}$ ESO: $\mathrm{N}=419$; $4^{\circ}$ ESO: $\mathrm{N}=366$; $1^{\circ}$ Ciencias y Tecnología: $\mathrm{N}=253$; $1^{\circ}$ Humanidades y Ciencias Sociales: $\mathrm{N}=253 ; 2^{\circ}$ Ciencias y Tecnología; $\mathrm{N}=90 ; 2^{\circ}$ Humanidades y Ciencias Sociales: $\mathrm{N}=106$.

a Se identifica con * el grupo con mayor promedio cuando las diferencias son significativas.

b Obtenido para las diferencias significativas. 
En primer lugar, las diferencias en autoeficacia a favor de los varones identificadas para el conjunto de la muestra no se encontraron cuando consideramos exclusivamente la modalidad de Ciencias y Tecnología. Esto es, chicas y chicos de esta modalidad presentaron niveles de autoeficacia científico-matemática equivalentes. Pero no es el caso durante la ESO o en el Bachillerato de Humanidades y Ciencias Sociales, donde los varones presentaron promedios siempre significativamente superiores. Las diferencias son, en general, de baja magnitud, siendo las más importantes las registradas al término del Bachillerato de Humanidades y Ciencias Sociales.

En lo que se refiere a las expectativas de resultado, las modestas diferencias encontradas en la muestra global de hecho se reducen a un ligero mayor promedio de los chicos en $3^{\text {o }}$ de ESO. Por el contrario, los mayores promedios en la medida de intereses a favor de los chicos se registraron en todos los cursos y modalidades con una sola excepción: $1^{\underline{o}}$ de Bachillerato de Ciencias, donde ambos presentaron promedios equivalentes. Aunque la magnitud de las diferencias es de nuevo modesta, destacan las correspondientes al término de la ESO y al término del Bachillerato en Ciencias y Tecnología. En lo que se refiere a las aspiraciones ocupacionales, sólo encontramos una diferencia muy modesta a favor de las chicas en $1^{\circ}$ de Ciencias.

Finalmente, las diferencias globales encontradas a favor de las chicas en la percepción de apoyo social se concentraron, particularmente, en $4^{\underline{0}}$ de ESO y en $1^{\circ}$ de Bachillerato de ambas modalidades. La magnitud de las diferencias es, de nuevo, muy modesta.

\section{Análisis de las diferencias en las variables sociocognitivas asociadas al curso}

Para todas las variables se registraron diferencias significativas asociadas al curso cuando se consideró la muestra total (tabla 4). Este patrón de resultados es común al obtenido en las submuestras de mujeres y varones, con dos excepciones: en autoeficacia y en apoyos y barreras sociales no se registraron diferencias significativas en la submuestra de mujeres en función del curso. La tendencia descriptiva de los promedios apunta en todo caso a menores niveles en todas las variables a medida que se avanza de curso, salvo en el caso de los apoyos y barreras percibidos, aparentemente más estables.

Identificada la tendencia general de los datos, se procedió a realizar las comparaciones múltiples posteriores que permitieran determinar con mayor matiz la generalidad del decremento en los promedios a medida que avanzan los cursos (ver tabla 5). Para los varones, el principal decremento en los promedios de autoeficacia, expectativas de resultados e intereses se registró entre $3^{\mathrm{o}}$ de ESO y Bachillerato. En el caso de las mujeres, donde no se registró disminución significativa en autoeficacia, los intereses sí registran el mismo patrón de disminución que los varones. También son similares, con algún matiz, las diferencias halladas en las mujeres para las expectativas de resultado: sus promedios fueron significativamente más elevados en $3^{\text {o- }} 4^{\text {o }}$ de ESO que al término del Bachillerato. Las diferencias encontradas son muy modestas, aunque algo más elevadas en lo que respecta al decremento de los varones en las expectativas de resultados en el área científico-matemática entre el inicio del segundo ciclo de ESO y Bachillerato. 
Igualmente se ha hallado una disminución estadísticamente significativa en los promedios correspondientes a las aspiraciones ocupacionales entre $3^{\circ}$ de ESO y Bachillerato, en ambos sexos. Para los varones se registró además un descenso significativo entre $3^{\mathrm{o}}$ y $4^{\mathrm{o}}$ de ESO y entre $4^{\mathrm{o}}$ y $1^{\mathrm{o}}$ de Bachillerato. Por su parte, las mujeres presentaron un descenso significativo entre $4^{\circ}$ de ESO y $2^{\circ}$ de Bachillerato.

Tabla 4

Comparación de los niveles promedio según el curso

\begin{tabular}{|c|c|c|c|c|c|c|c|c|c|}
\hline & \multicolumn{3}{|c|}{ Muestra completa } & \multicolumn{3}{|c|}{ Mujeres } & \multicolumn{3}{|c|}{ Varones } \\
\hline & Mediana & $\chi^{2}$ & $p$ & Mediana & $\chi^{2}$ & $\mathrm{p}$ & Mediana & $\chi^{2}$ & $\mathrm{p}$ \\
\hline \multicolumn{10}{|l|}{ Autoeficacia } \\
\hline $3^{\circ} \mathrm{ESO}$ & 4.67 & \multirow{4}{*}{14.89} & \multirow{4}{*}{.002} & 4.58 & \multirow{4}{*}{5.27} & \multirow{4}{*}{.153} & 4.81 & \multirow{4}{*}{8.99} & \multirow{4}{*}{.029} \\
\hline $4^{\mathrm{o}} \mathrm{ESO}$ & 4.50 & & & 4.37 & & & 4.62 & & \\
\hline $1^{\circ}$ Bachillerato & 4.50 & & & 4.33 & & & 4.58 & & \\
\hline 2ํㅡachillerato & 4.32 & & & 4.19 & & & 4.50 & & \\
\hline \multicolumn{10}{|c|}{$\begin{array}{l}\text { Expectativas de } \\
\text { resultado }\end{array}$} \\
\hline 3ํESO & 5.67 & \multirow{4}{*}{33.19} & \multirow{4}{*}{.000} & 5.55 & \multirow{4}{*}{9.53} & \multirow{4}{*}{.023} & 5.78 & \multirow{4}{*}{24.93} & \multirow{4}{*}{.000} \\
\hline $4^{\mathrm{o}} \mathrm{ESO}$ & 5.39 & & & 5.33 & & & 5.44 & & \\
\hline $1^{\circ}$ Bachillerato & 5.22 & & & 5.22 & & & 5.22 & & \\
\hline $2^{\circ}$ Bachillerato & 5.11 & & & 5.11 & & & 5.22 & & \\
\hline \multicolumn{10}{|l|}{ Intereses } \\
\hline 3트O & 4.62 & \multirow{4}{*}{38.02} & \multirow{4}{*}{.000} & 4.56 & \multirow{4}{*}{20.73} & \multirow{4}{*}{.000} & 4.78 & \multirow{4}{*}{15.99} & \multirow{4}{*}{.001} \\
\hline $4^{\circ} \mathrm{ESO}$ & 4.50 & & & 4.25 & & & 4.81 & & \\
\hline $1^{\circ}$ Bachillerato & 4.34 & & & 4.25 & & & 4.50 & & \\
\hline $2^{\circ}$ Bachillerato & 4.06 & & & 4.06 & & & 4.31 & & \\
\hline \multicolumn{10}{|l|}{ Aspiraciones } \\
\hline $3^{\circ} \mathrm{ESO}$ & 3.50 & \multirow{4}{*}{73.83} & \multirow{4}{*}{.000} & 3.50 & \multirow{4}{*}{45.45} & \multirow{4}{*}{.000} & 3.60 & \multirow{4}{*}{33.44} & \multirow{4}{*}{.000} \\
\hline $4^{\circ} \mathrm{ESO}$ & 3.20 & & & 3.20 & & & 3.15 & & \\
\hline 1ํBachillerato & 2.80 & & & 2.90 & & & 2.70 & & \\
\hline $2^{\circ}$ Bachillerato & 2.60 & & & 2.50 & & & 3.00 & & \\
\hline $\begin{array}{l}\text { Apoyos y barr } \\
\text { sociales }\end{array}$ & & & & & & & & & \\
\hline 3ESO & 6.00 & & & 5.87 & & & 6.00 & & \\
\hline $4^{\circ} \mathrm{ESO}$ & 5.75 & 9.94 & .019 & 5.87 & 0.56 & .906 & 5.50 & 16.98 & .001 \\
\hline $1^{\circ}$ Bachillerato & 5.75 & & & 6.00 & & & 5.50 & & \\
\hline $2^{\circ}$ Bachillerato & 5.87 & & & 5.87 & & & 5.75 & & \\
\hline
\end{tabular}


Tabla 5

Comparaciones post hoc en función del curso y tamaño del efecto

\begin{tabular}{|c|c|c|c|c|c|}
\hline \multirow{2}{*}{ Comparación } & \multicolumn{3}{|c|}{ Muestra completa } & \multirow{2}{*}{$\frac{\text { Mujeres }^{\mathrm{a}}}{\mathrm{r}}$} & \multirow{2}{*}{$\frac{\text { Varones }^{\mathrm{a}}}{\mathbf{r}}$} \\
\hline & $\mathrm{U}$ & $\mathrm{p}$ & $\mathbf{r}$ & & \\
\hline \multicolumn{6}{|l|}{ Autoeficacia } \\
\hline $3^{\circ} \mathrm{ESO}-4^{\circ} \mathrm{ESO}$ & 71400.50 & .096 & - & - & - \\
\hline $3^{\circ}$ ESO - 1ํㅡachillerato & 92781.50 & .001 & 0.11 & - & 0.13 \\
\hline $3^{\circ} \mathrm{ESO}-2^{\circ}$ Bachillerato & 34603.00 & .002 & 0.13 & - & - \\
\hline $4^{\circ} \mathrm{ESO}-1^{\circ}$ Bachillerato & 87020.50 & .129 & - & - & - \\
\hline $4^{\circ} \mathrm{ESO}-2^{\circ}$ Bachillerato & 32605.00 & .075 & - & - & - \\
\hline $1^{\circ}$ Bachillerato $-2^{\circ}$ Bachillerato & 48356.50 & .609 & - & - & - \\
\hline \multicolumn{6}{|l|}{ Expectativas de resultado } \\
\hline $3^{\circ} \mathrm{ESO}-4^{\circ} \mathrm{ESO}$ & 67074.50 & .002 & 0.11 & - & - \\
\hline $3^{\circ} \mathrm{ESO}-1^{\circ}$ Bachillerato & 86725.00 & .000 & 0.16 & - & 0.21 \\
\hline $3^{\circ} \mathrm{ESO}-2^{\circ}$ Bachillerato & 31196.50 & .000 & 0.19 & 0.15 & 0.21 \\
\hline $4^{\circ} \mathrm{ESO}-1^{\circ} \mathrm{B}$ Bachillerato & 86171.50 & .080 & - & - & - \\
\hline $4^{\circ} \mathrm{ESO}-2^{\circ} \mathrm{B}$ Bachillerato & 31304.50 & .013 & - & 0.09 & - \\
\hline $1^{\circ}$ Bachillerato $-2^{\circ}$ Bachillerato & 47098.00 & .301 & - & - & - \\
\hline \multicolumn{6}{|l|}{ Intereses } \\
\hline $3^{\circ} \mathrm{ESO}-4^{\circ} \mathrm{ESO}$ & 69063.00 & .016 & - & - & - \\
\hline $3^{\circ} \mathrm{ESO}-1^{\circ}$ Bachillerato & 87951.50 & .000 & 0.15 & 0.14 & 0.15 \\
\hline $3^{\circ}$ ESO - 2º Bachillerato & 29415.50 & .000 & 0.23 & 0.24 & 0.18 \\
\hline $4^{\mathrm{o}} \mathrm{ESO}-1^{\mathrm{o}}$ Bachillerato & 85828.50 & .065 & - & - & - \\
\hline $4^{\circ} \mathrm{ESO}-2^{\circ}$ Bachillerato & 29206.50 & .000 & 0.15 & - & - \\
\hline $1^{\circ}$ Bachillerato $-2^{\circ}$ Bachillerato & 44309.50 & .029 & - & - & - \\
\hline \multicolumn{6}{|l|}{ Aspiraciones ocupacionales } \\
\hline $3^{\circ} \mathrm{ESO}-4^{\circ} \mathrm{ESO}$ & 65643.50 & .000 & 0.12 & - & 0.14 \\
\hline $3^{\circ}$ ESO - 1ํachillerato & 76494.50 & .000 & 0.24 & 0.22 & 0.26 \\
\hline $3^{\circ}$ ESO - 2º Bachillerato & 26728.50 & .000 & 0.28 & 0.35 & 0.18 \\
\hline $4^{\circ} \mathrm{ESO}-1^{\circ}$ Bachillerato & 79955.50 & .001 & 0.12 & - & 0.13 \\
\hline $4^{\circ} \mathrm{ESO}-2^{\circ} \mathrm{o}$ Bachillerato & 28465.00 & .000 & 0.17 & 0.23 & - \\
\hline $1^{\circ}$ Bachillerato $-2^{\circ}$ Bachillerato & 46155.50 & .154 & - & - & - \\
\hline \multicolumn{6}{|l|}{ Apoyos y barreras sociales } \\
\hline $3^{\circ} \mathrm{ESO}-4^{\circ} \mathrm{ESO}$ & 67203.50 & .003 & 0.11 & - & 0.18 \\
\hline $3^{\circ} \mathrm{ESO}-1^{\circ}$ Bachillerato & 96348.50 & .017 & - & - & 0.16 \\
\hline $3^{\circ} \mathrm{ESO}-2^{\circ}$ Bachillerato & 37708.50 & .102 & - & - & - \\
\hline $4^{\circ} \mathrm{ESO}-1^{\circ} \mathrm{o}$ Bachillerato & 89753.00 & .438 & - & - & - \\
\hline $4^{\circ} \mathrm{ESO}-2^{\circ}$ Bachillerato & 34572.00 & .480 & - & - & - \\
\hline $1^{\mathrm{o}}$ Bachillerato $-2^{\circ}$ Bachillerato & 49288.00 & .901 & - & - & - \\
\hline
\end{tabular}

Nota: Se ajustan los niveles de alpha mediante el procedimiento de Bonferroni a 0.008 para cada test $(0.05 / 6)$.

a Obtenido para las diferencias estadísticamente significativas. 
Aunque las diferencias registradas son de magnitud muy discreta, conviene destacar que el tamaño del efecto asociado al decremento en las aspiraciones ocupaciones en el área científico-matemático de las mujeres entre $3^{\circ}$ de ESO y $2^{\circ}$ de Bachillerato es de los mayores de este estudio. También conviene destacar que tanto para varones como para mujeres no se identificaron diferencias en las aspiraciones ocupacionales entre los dos cursos de Bachillerato.

Finalmente, los promedios en apoyos y barreras sociales percibidos, que para las mujeres no mostraron diferencias significativas a lo largo de los cuatro cursos evaluados, presentaron un ligero descenso para los varones entre $3^{\circ}$ y $4^{\circ}$ de ESO $/ 1^{\circ}$ de Bachillerato.

\section{Discusión y conclusiones}

El objetivo de este estudio fue analizar, desde la perspectiva de la SCCT, las posibles diferencias existentes entre alumnas y alumnos de secundaria en varios factores relevantes en la elección vocacional en el área científico-matemática. Este enfoque diferencial tomó en consideración el curso y la modalidad de estudio, con el fin de proporcionar una visión más matizada no llevada a cabo hasta ahora con alumnado español.

En primer lugar, llevamos a cabo un análisis preliminar de la sensibilidad de las medidas. Conforme a lo esperado, los resultados sugirieron que las medidas sociocognitivas empleadas son válidas en términos de capacidad de discriminación entre grupos distintos (Ciencias y Tecnología versus Humanidades y Ciencias Sociales).

En cuanto a los objetivos principales planteados, los resultados se alinean con los informados en la literatura sobre diferencias a favor de los varones cuando se comparan sus expectativas (de autoeficacia y de resultados) e intereses en el área STEM. También nuestros resultados apoyan el decremento paulatino de las actitudes en el ámbito científico-matemático informado por la investigación previa. Aunque las magnitudes de las diferencias encontradas a lo largo del estudio son muy modestas.

Efectivamente los chicos presentaron un promedio significativamente más alto que las chicas en autoeficacia, intereses y expectativas de resultados. Estos resultados concuerdan con los obtenidos por Bandura, Barbaranelli, Caprara y Pastorelli (2001), Navarro et al. (2007) y O'Brien et al. (1999) en lo referido a la autoeficacia; Turner y Lapan (2005) en lo que respecta a los intereses; o Fouad y Smith (1996) en cuanto a las expectativas de resultado. Así, nuestros datos no pueden confirmar el patrón de equivalencia informado por Rodríguez et al. (2016) para estas mismas variables con alumnado de secundaria español en el área de la tecnología. Pero más allá de este patrón general, algunos resultados más específicos pueden ser de especial interés.

En nuestro estudio no se encontraron diferencias en autoeficacia entre chicas y chicos de la modalidad de Ciencias y Tecnología, lo que confirma la importancia de considerar esta variable en el análisis, y apoya parcialmente la hipótesis de un cambio de tendencia en las expectativas de las alumnas, apuntada por Rodríguez et al. (2016). En todo caso, la desagregación permite identificar cómo las mujeres durante el segundo ciclo de ESO, momento clave en la toma de decisiones académico-profesio- 
nales, presentan menores niveles de autoeficacia científico-matemática. Pero también al término del Bachillerato de Humanidades y Ciencias Sociales sus percepciones de competencia científico-matemática son menores que las de sus compañeros varones de modalidad. Esto podría colocarlas en una situación de desventaja en sus trayectorias de elección ligada a unas percepciones de autoeficacia sesgadas o imprecisas, lo que señala una posible línea de intervención necesaria. Conviene notar que, frente al descenso en las percepciones de autoeficacia que se registran en los varones entre el inicio del segundo ciclo de ESO y Bachillerato, en las chicas permanecen estables.

Menos claras son las diferencias entre varones y mujeres encontradas sobre las expectativas de resultado, pues están acotadas en un solo curso de los evaluados: $3^{\mathrm{o}}$ de ESO. En todo caso, puesto que las percepciones de utilidad y valor están relacionadas con el desarrollo de intereses en un ámbito dado, podría ser importante promover en las alumnas expectativas realistas y basadas en información veraz sobre el ámbito científico-matemático en el arranque del segundo ciclo de ESO. Además, puesto que para varones y mujeres se registra un cierto deterioro de las expectativas entre ESO y Bachillerato, los resultados sugieren que las intervenciones deberían desarrollarse tempranamente.

También en la línea de otros trabajos, en el estudio se obtuvieron mayores promedios en los intereses expresados por los varones que por las mujeres. Estas diferencias fueron mayores al término de ESO, pero también al término del Bachillerato de Ciencias y Tecnología, lo que parece indicar la necesidad de considerar a las "alumnas de ciencias" como un colectivo para el que podría ser necesario también el fomento del interés por trayectorias en el ámbito científico-matemático.

En lo que se refiere a los apoyos y barreras sociales percibidos, frente a lo hallado por Rodríguez et al. (2016), las mujeres puntuaron significativamente más alto. Esto es, percibieron en mayor medida que los varones que una posible decisión vocacional en el ámbito científico-matemático sería positivamente apoyada por su entorno social más próximo (familia, compañeros y amigos, escuela). Estos resultados son, por lo demás, coincidentes con los informados por Navarro et al. (2007) o Santana et al. (2012). Ellas se sienten más apoyadas en dos momentos importantes en la definición de la trayectoria académico-profesional: $4^{\circ}$ de ESO y $1^{\text {o }}$ de Bachillerato de ambas modalidades. Sus percepciones son estables a lo largo de los cuatro cursos evaluados, mientras que para los varones sufren un ligero descenso entre ESO y el Bachillerato.

Finalmente, no se encontraron diferencias entre chicos y chicas en aspiraciones ocupacionales. Además, chicos y chicas comparten globalmente un patrón de descenso entre ESO y el Bachillerato, algo más acentuado para las mujeres. Este resultado, por un lado, contrasta con la evidencia ampliamente documentada de que los varones predominan en la selección de opciones laborales científicas, matemáticas o técnicas (Alloza et al., 2011; Canto, 2000; Rodríguez et al., 2014; Rodríguez et al 2012). Por otro, sugiere que la selección final de metas/opciones pueden estar sólo parcialmente mediada por las aspiraciones, entendidas como una expresión de intenciones más o menos vaga. En todo caso parece claro que se trata de un resultado que necesita ser confirmado con nuevas muestras y también con medidas alternativas. 
Tomados en conjunto, los resultados de este estudio creemos que hacen una aportación relevante a los trabajos desarrollados en España sobre trayectorias vocacionales en el área STEM desde el ámbito psicopedagógico. Sin embargo, los hallazgos descritos deben ser entendidos en el marco de algunas de las limitaciones de la presente investigación. En primer lugar, si bien el tamaño de la muestra y su composición dotan a los resultados de una fiabilidad razonable, su selección no ha sido aleatoria y por tanto la generalización de estos resultados debería hacerse con cautela. En segundo lugar, dado el carácter transversal del diseño, el análisis de la evolución de las expectativas, intereses, percepciones y aspiraciones del alumnado a lo largo de los cursos tiene un carácter naturalmente limitado. En tercer lugar, un análisis más completo de los factores sociocognitivos relevantes en la etapa estudiada debería haber incluido cursos no considerados. Por tanto, sería relevante desarrollar en el futuro estudios longitudinales cuyo inicio tenga lugar en $1^{\circ}$ de ESO. Ello permitiría tener una visión más amplia y dinámica de los procesos de desarrollo de intereses y elecciones académico-profesionales en el área STEM en general y científico-matemática en particular.

\section{Referencias}

Alloza, F. M., Anghel, B., Dolado, J. J., De la Rica, S., \& Sánchez de Madariaga, I. (2011). Libro Blanco: Situación de las mujeres en la ciencia española. Madrid: Ministerio de Ciencia e Innovación.

Bandura, A., Barbaranelli, C., Caprara, G. V., \& Pastorelli, C. (2001). Self-efficacy beliefs as shapers of children's aspirations and career trajectories. Child development, 72(1), 187-206. doi:10.1111/1467-8624.00273.

Blanco, A. (2011). Applying social cognitive career theory to predict interests and choice goals in statistics among Spanish psychology students. Journal of Vocational Behavior, 78, 49-58. doi: 10.1016/j.jvb.2010.07.003.

Blanco-Blanco, A., Casas, Y., \& Mafokozi, J. (2016). Adaptación y propiedades psicométricas de escalas sociocognitivas. Una aplicación en el ámbito vocacional científico-matemático. Revista Española de Orientación y Psicopedagogía, 27(1), 8-28. doi: 10.5944/reop.vol.27.num.1.2016.17005.

Byars-Winston, A., Estrada, Y., Howard, C., Davis, D., \& Zalapa, J. (2010). Influence of social cognitive and ethnic variables on academic goals of underrepresented students in science and engineering: A multiple-groups analysis. Journal of Counseling Psychology, 57(2), 205-218. doi:10.1037/a0018608.

Canto, J. E. (2000). Certeza de elección de carrera y preferencia vocacional. Educación y Ciencia, 4(7), 43-55. Recuperado de

educacionyciencia.org/index.php/educacionyciencia/article/download/136/pdf

Cohen, J. (1988). Statistical power analysis for the behavioral sciences (2a ed.). Hillsdale, New Jersey: Erlbaum.

Corder, G. W., \& Foreman, D. I. (2009). Non parametric Statistics for non-statistician. A step-by-step approch. Hoboken, New Jersey: John Wiley \& Sons. 
Centro para el Desarrollo Técnico Industrial-CDTI (2014). Guía Horizonte 2020. Madrid: Autor.

Flores, L., \& O'Brien, K. (2002). The career development of Mexican American adolescent women: a test of social cognitive career theory. Journal of Counseling Psychology, 49(1), 14-27. doi:10.1037/0022- 0167.49.1.14.

Fouad, N. A., \& Smith, P. L. (1996). A test of a social cognitive model for middle school students: Math and science. Journal of Counseling Psychology, 43(3), 338-346. doi:10.1037/0022-0167.43.3.338.

Garriot, P.O., Flores, L.Y., \& Martens, M.P. (2013). Predicting the math/science career goals of low-income prospective first-generation college students. Journal of Counseling Psychology, 60(2), 200-209. doi:10.1037/a0032074.

Hidalgo, S. A., Maroto, A. S., \& Palacios, A. P. (2004). ¿Por qué se rechazan las matemáticas? Análisis evolutivo y multivariante de actitudes relevantes hacia las matemáticas. Revista de Educación, 334, 75-95. Recuperado de http://www.revistaeducacion.mec.es/re334_06.htm

Lent, R. W., Brown, S. D., \& Hackett, G. (1994). Toward a unifying social cognitive theory of career and academic interest, choice, and performance. Journal of Vocational Behavior, 45(1), 79-122. doi: 10.1006/jvbe.1994.1027.

Lent, R. W., Brown, S. D., \& Hackett, G. (2000). Contextual supports and barriers to career choice: a social cognitive analysis. Journal of Counseling Psychology, 47(1), 36-49. doi: 10.1037//0022-0167.47.1.36.

Lent, R. W., Brown, S. D., Brenner, B., Chopra, S. B., Davis, T., Talleyrand, R., \& Sthakaran, V. (2001). The role of contextual supports and barriers in the choice of math/science educational options: A test of social cognitive career hypotheses. Journal of Counseling Psychology, 48(4), 474-483. doi: 10.1037//0022-0167.48.4.474

Lent, R. W., Sheu, H., Gloster, C. S., \& Wilkins, G. (2010). Longitudinal test of social cognitive model of choice in engineering students at historically black universities. Journal of Vocational Behavior, 76(3), 387-394. doi:10.1016/j.jvb.2009.09.002.

Lent, R. W., Sheu, H., Singley, D., Schmidt, J., Schmidt, L., \& Gloster, C. (2008). Longitudinal relations of self-efficacy to outcome expectations, interest, and major choice goals in engineering students. Journal of Vocational Behavior, 73(2), 328-335. doi: 10.1016/j.jvb.2008.07.005.

Navarro, R.L., Flores, L.Y., \& Worthington, R.L. (2007). Mexican American middle school students' goal intentions in mathematics and science: a test of social cognitive career theory. Journal of Counseling Psychology, 54(3), 320-335. doi:10.1037/00220167.54.3.320.

Núñez, J. C., González-Pienda, J. A., Alvarez, L., González-Castro, P., GonzálezPumariega, S., Roces, C., ... \& Da Silva, E. H. (2005). Las actitudes hacia las matemáticas: perspectiva evolutiva. En Actas do VIII Congreso Galaico-Portugués de Psicopedagoxía, 2389-2396. Obtenido de http://www.educacion.udc.es/grupos/ gipdae/documentos/congreso/viiicongreso/pdfs/291.pdf.

O’Brien, V., Martínez-Pons, M., \& Kopola, M. (1999). Mathematics self-efficacy, ethnic identity, gender and career interests related to mathematics and science. The Journal of Educational Research, 92(4), 231-235. doi:10.1080/00220679909597600. 
OECD (2008). Encouraging Student Interest in Science and Technology Studies. Paris: OECD.

Rodríguez C., Inda, M., \& Fernández, M.C. (2016). Influence of social cognitive and gender variables on technological academic interest among Spanish high-school students: testing social cognitive career theory. International Journal for Educational and Vocational Guidance,16 (3), 305-325. doi: 10.1007/s10775-015-9312-8.

Rodríguez, M. C., Peña, J. V., \& Inda, M. M. (2012). Creencias de autoeficacia y elección femenina de estudios científico-tecnológicos. Teoría de la Educación, 24(1), 81-104. Recuperado de gredos.usal.es/jspui/bitstream/10366/131084/1/Creencias_ de_autoeficacia_y_eleccion_fem.pdf

Rodríguez, M. C., Inda, M. M., \& Peña, J. V. (2014). Rendimiento en la PAU y elección de estudios científico-tecnológicos en razón de género. Revista Española de Orientación y Psicopedagogía, 25(1), 111-127. doi: 10.5944/reop.vol.25.num.1.2014.12016.

Rodríguez, M. C., Inda, M. M., \& Peña, J. V. (2015). Validación de la Teoría Cognitivo Social de Desarrollo de Carrera con una muestra de estudiantes de ingeniería. Educación XX1, 18(2), 257-276. doi:10.5944/educXX1.14018

Rodríguez, M. C., Torío, L. S., \& Fernández, G. C. M. (2006). El impacto del género en las elecciones académicas de los estudiantes asturianos que finalizan la ESO. Revista Española de Orientación y Psicopedagogía, 2(2), 239-260. doi: 10.5944/reop. vol.17.num.2.2006.11351.

Rovai, A. P., Baker, J. D., \& Ponton, M. (2014). Social Science Research Design and Statistics. A practitioner's guide to research methods and IMB SPSS analysis (2a ed.). Chesapeake, Virginia: Watertree Press LLC.

Santana, L. E., Feliciano, L., \& Jiménez, A. B. (2012). Toma de decisiones y género en el bachillerato. Revista de Educación, 359, 357-387. doi:10.4438/1988592X-RE-2011-359-098.

Sheu, H., \& Bordon, J. (2017). SCCT research in the international context: empirical evidence, future directions, and practical implications. Journal of Career Assessment, 25(1), 58-74. doi: 10.1177/1069072716657826

Sheu, H., Lent, R. W., Brown, S., Miller, M., Hennessy, K., \& Duffy, R. D. (2010). Testing the choice model of social cognitive career theory across Holland themes: A meta-analytic path analysis. Journal of Vocational Behavior, 76(2), 252-264. doi 10.1016/j.jvb.2009.10.015.

Turner, S. L., \& Lapan, R. T. (2005). Evaluation of an intervention to increase non-traditional career interests and career-related self-efficacy among middle-school adolescents. Journal of Vocational Behavior, 66(3), 516-531. doi:10.1016/j.jvb.2004.02.005.

Vázquez, A., \& Manassero, M. A. (2005). La ciencia escolar vista por los estudiantes. Bordón: Revista de Pedagogía, 57(5), 717-736. Recuperado de https://recyt.fecyt.es/ index.php/BORDON/article/view/40802

Vázquez, A., \& Manassero, M. A. (2008). El declive de las actitudes hacia la ciencia de los estudiantes: un indicador inquietante para la educación científica. Revista Eureka sobre Enseñanza y Divulgación de las Ciencias, 5(3), 274-292. Recuperado de https://revistas.uca.es/index.php/eureka/article/view/3740 
Vázquez, A., \& Manassero, M. A. (2009). Patrones actitudinales de la vocación científica y tecnológica en chicas y chicos de secundaria. Revista Iberoamericana de Educación, 50(4), 1-15. Recuperado de: https://rieoei.org/RIE/article/view/1879

Vázquez, A., \& Manassero, M. A. (2015). La elección de estudios superiores científicotécnicos: análisis de algunos factores determinantes en seis países. Revista Eureka sobre Enseñanza y Divulgación de las Ciencias, 12(2), 264-277. doi: 10498/17251.

Wang, M., \& Degol, J. (2013). Motivational Pathways to STEM Career Choices: Using Expectancy-Value Perspective to Understand Individual and Gender Differences in STEM Fields. Developmental Review, 33(4), 304-340. doi: 10.1016/j.dr.2013.08.001.

Wang, X. (2013). Why students choose STEM majors: motivation, high school learning, and postsecondary context of support. American Educational Research Journal, 50(5), 1081-1121. doi:10.3102/0002831213488622.

Fecha de recepción: 4 de septiembre de 2017.

Fecha de revisión: 19 de septiembre de 2017.

Fecha de aceptación: 19 de febrero de 2018. 